\title{
Armin Schoklitsch: Wasserbau-Karriere in Brünn (Teil 2)
}

\author{
Willi H. Hager
}

Online publiziert: 21. März 2015

(C) Springer-Verlag Wien 2015

Zusammenfassung Das Werk und Leben von Armin Schoklitsch von seiner Ernennung im Jahr 1926 zum WasserbauProfessor an der Deutschen Technischen Hochschule in Brünn über die Rückkehr an die TH Graz bis zur Übersiedlung nach Argentinien wird beschrieben. In diesem Zeitabschnitt entstanden verschiedene wichtige Bücher des Wasser- und Flussbaus, die im Lichte der Entwicklungen dieser Fachgebiete erläutert werden. Der Einfluss der Forschungen Schoklitschs insbesondere auf den Sedimenttransport wird dabei beleuchtet. Weiterhin soll das Leben des Wasserbau-Ingenieurs in den Zwischenkriegsjahren beschrieben werden. Schließlich wird Schoklitschs Rolle in Graz während der letzten Kriegsjahre besprochen.

\section{Armin Schoklitsch: Hydraulic engineering career in Brno (Part 2)}

Abstract The work and life of Armin Schoklitsch from his appointment in 1926 as professor of hydraulic engineering at the German Technical University of Brno over his return to TH Graz to his emigration to Argentina in 1948 is described. During this era, many of his important writings in hydraulic and river engineering were published, which are reviewed in terms of the corresponding developments. Schoklitsch's impact on sediment transport in particular is highlighted and compared with parallel scientific developments. The life and career spots of this hydraulic engineer in the 1930s are further highlighted. Finally, Schoklitsch's role at TH Graz during the last war years is described.

Prof. Dr. W. H. Hager $(\bowtie)$

Versuchsanstalt für Wasserbau, Hydrologie und Glaziologie (VAW), Eidgenössische Technische Hochschule Zürich, Wolfgang-Pauli-Str. 27, 8093 Zürich, Schweiz

E-Mail: hager@vaw.baug.ethz.ch

\section{Einleitung}

Der Werdegang zum Wasserbau-Ingenieur und die Forschungsbeiträge von Armin Schoklitsch (1888-1969, Abb. 1) wurden im 1. Teil dieser Arbeit besprochen (Hager 2015). Es wurde festgehalten, dass es sich bei ihm um einen hervorragenden Hydrauliker und Wasserbau-Ingenieur handelte, der durch seinen Lehrer Philipp Forchheimer (1852-1933) gefördert wurde. Nachdem Schoklitsch 1913 seine Promotionsarbeit erfolgreich eingereicht hatte, war er bis 1918 praktisch ununterbrochen am 1. Weltkrieg beteiligt, kehrte dann als Privatdozent an die Technische Hochschule (TH) Graz zurück und baute sich ein beachtliches hydraulisches Labor auf. Gleichzeitig war er auch mit der Lehre und wasserbaulichen Aufträgen beschäftigt. Trotz dieses vielfältigen Arbeitsprogramms fand er Zeit für eine für damalige Verhältnisse große Publikations-Tätigkeit. Ab 1926 war Schoklitsch Professor für Wasserbau und Grundbau an der Deutschen TH Brünn in der Tsche-

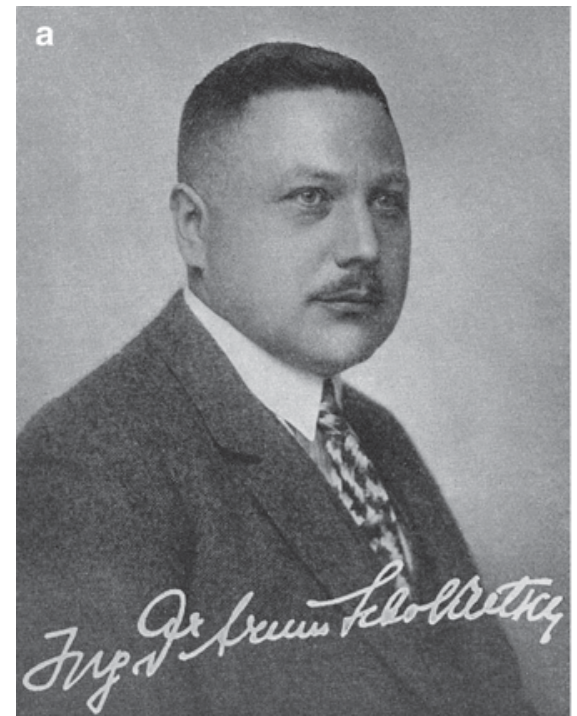

Abb. 1 Armin Schoklitsch a) um 1926 (Foto: Schoklitsch 1929a, Quelle: Pietschmann 1999), b) um 1930 (Foto: Pietschmann, Quelle: Pietschmann 1999)

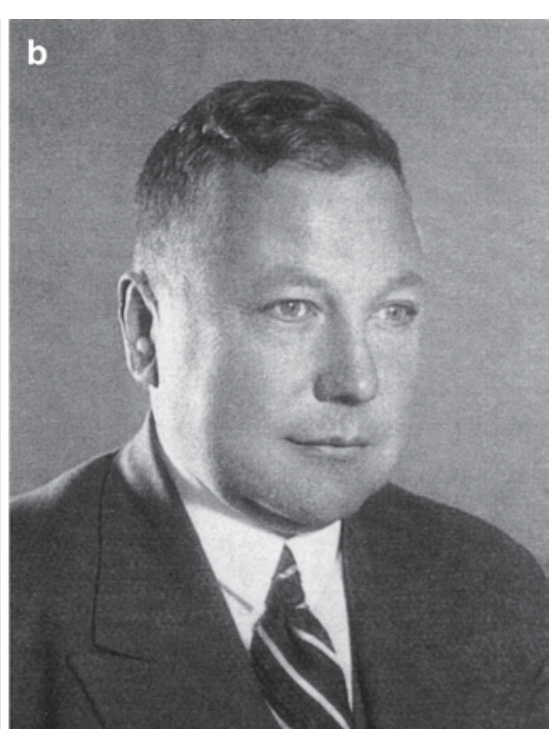

choslowakei, wo er sich zum eigentlichen Wasserbau-Ingenieur entwickelte. Es entstand eine Anzahl außerordentlicher Werke, die nachfolgend vorgestellt werden sollen. Der Zeitrahmen erstreckt sich dabei bis 1949, dem Jahr seiner Übersiedlung nach Argentinien. Damit soll eine wichtige Persönlichkeit des Wasserbaus der Zwischenkriegszeit gebührend geehrt und sein Werk beschrieben werden.

\section{Publikationen}

Wie bereits in Teil 1 festgehalten, beschäftigte sich Schoklitsch neben dem Wasserbau auch intensiv mit dem Grundbau und der Bodenmechanik. Dies folgt etwa aus seiner Arbeit zur Durchlässigkeit von Baumaterialien, welche noch 1923 in seinem Abdichtungslaboratorium an der $\mathrm{TH}$ Graz durchgeführt wurde (Schoklitsch 1926a). So wurden vorerst Betonproben zwischen zwei Rundgummidichtungen in einen Apparat eingespannt, welche von oben her mit Druckwasser beaufschlagt wurden, das dann die Probe durchfloss 
und seitlich, resp. unten, wieder austrat. Üblicherweise ließ sich bei allen Proben, die nicht von Anfang an dicht waren, eine langsam fortschreitende Selbstdichtung beobachten. Bei rund 4 atü Wasserdruck hörte die Durchsickerung nach einigen Monaten komplett auf. Der Vorgang wurde mittels des Gesetzes von Darcy geklärt, nach dem die mittlere Durchsickerungs-Geschwindigkeit nur von einem Durchlässigkeits-Faktor und dem Drucklinien-Gefälle abhängt. Dieser Faktor variiert nur mit der Poren-Beschaffenheit und den Eigenschaften der durchsickernden Flüssigkeit. Es wurde vorerst nachgewiesen, dass es sich bei diesem Vorgang um laminares Fließen durch die Matrix handelt. Ähnliche Versuche wurden auch mit Holz und mit Sand durchgeführt. Die letzten Untersuchungen wurden in $1.4 \mathrm{~m}$ $(3.0 \mathrm{~m})$ langen Rohren von $0.20 \mathrm{~m}(0.50 \mathrm{~m})$ Durchmesser durchgeführt, in welche das zu untersuchende Material verdichtet eingebracht wurde; an einem Rohrende wurde Druckwasser eingeführt, das nach der Durchsickerung der Matrix entweder durch eine gelochte Platte frei austrat oder durch einen an der Abschlussplatte angeschlossenen Schlauch in ein Gefäß ausfloss (Abb. 2). Der Druckverlust entlang des Rohrs wurde durch Piezometer gemessen, womit sich Rückschlüsse auf das Verhalten des Materials bei Wasserbauten anstellen ließen. Die Resultate zeigten, dass der Durchlässigkeits-Faktor durch die Zusammensetzung des Sands und dessen Korngröße gegeben ist. Bei uniformem Sanddurchmesser bestätigt sich das Darcy-Gesetz bei laminarer Strömung, während sich ab etwa einer Geschwindigkeit von $0,005 \mathrm{~m} / \mathrm{s}$ turbulente Bewegung einstellt. Ähnliche Versuche wurden in der Folge erst ab etwa 1930 an verschiedenen hydraulischen Laboratorien durchgeführt, weshalb hier Schoklitsch wiederum als einer der Begründer der modernen Bodenmechanik bezeichnet werden darf.

Schoklitsch (1926b) verfasste eine rund 100 Seiten starke Abhandlung über die Geschiebebewegung, welche auf seine Zeit in Graz zurückgeht. Im Vorwort wird vermerkt, dass ,,in vieljähriger Arbeit Versuche und Beobachtungen über die Geschiebebewegung in Flussläufen, über die Aufbereitung von Geschiebe, die Verlandung der Stauräume, die Auswahl der Wasserfassungsstelle für ein Kraftwerk, die Bemessung des Einlaufes, den Einfluss der Tauchwand und eines Grobrechens auf die Verlandung des Einlaufbeckens, die Spülung des Staubereiches, die Sohlenausbildung

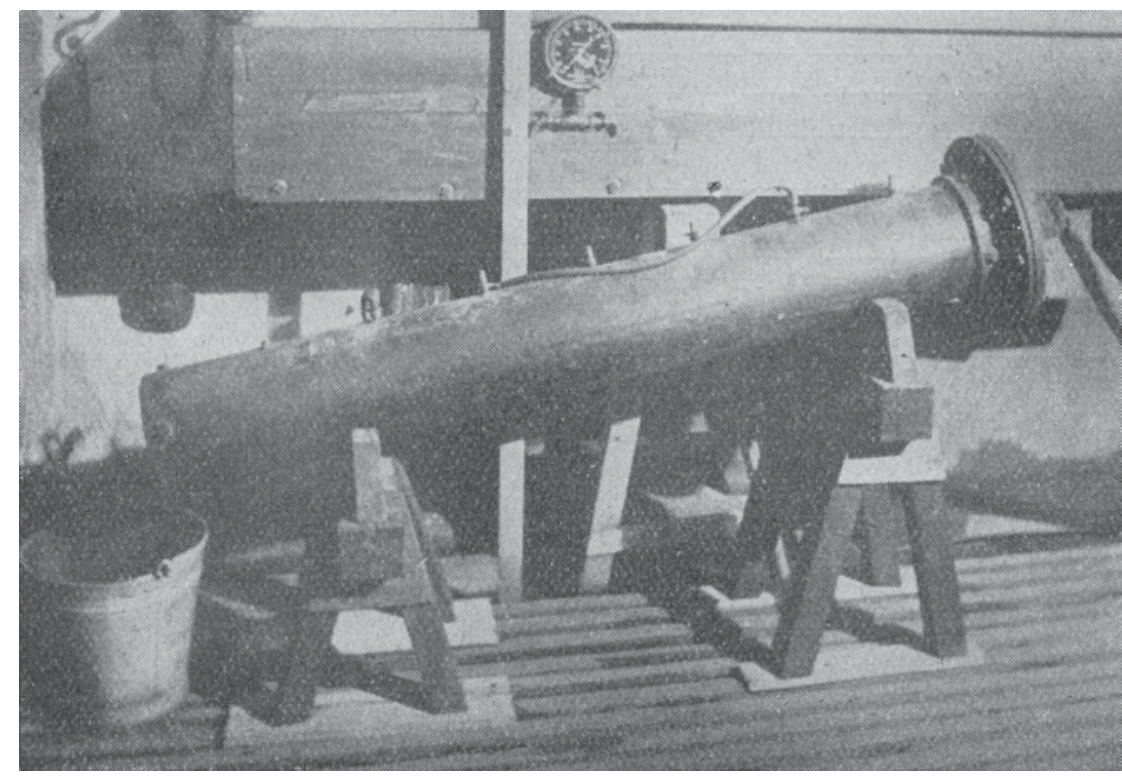

Abb. 2 Versuchsanordnung zur Ermittlung der Durchfluss-Eigenschaften von Sand unter hohem Zuflussdruck. (Schoklitsch 1926a)

unter dem Stauwerke und endlich über die Übereinstimmung der Ergebnisse von Modellversuchen mit den Vorgängen in der Natur" angestellt wurden. Vorerst wird Geschiebe geometrisch charakterisiert, dann dessen „Abnutzung“ erklärt sowie Entmischungsvorgänge an einer Sandbank vorgestellt. Die Sortierung von Geschiebe entlang einer Kurve wird erläutert, an welchen sich das grobe Geschiebe im Kolk längs der Außenkurve, das feine jedoch entlang einer Sandbank entlang der Innenkurve ablagert. Weiter im Unterwasser der Kurve verlagert sich der Stromstrich entlang der Innenkurve, mit einer entsprechenden Geschiebeumlagerung (Abb. 3). Es wird unterschieden zwischen Versuchen mit grobem Sand bis zum Durchmesser von $10 \mathrm{~mm}$ und feinem Sand bis zu $2 \mathrm{~mm}$. Weiter wird der Sortierungsprozess längs einer Kurve bildlich veranschaulicht. Die entstehende Sekundärströmung längs einer Kanalkurve und Geschiebeproben an diversen Querschnitten der Kurve werden beschrieben. Ähnliche Untersuchungen beziehen sich auch auf einen Kniekrümmer und eine Kanalabzweigung, woraus folgte, dass eine geschiebefreie Wasserentnahme aus einem Fluss längs der Bogenaußenseite zu erfolgen hat.

Kapitel 3 beschäftigt sich mit der Sinkstoffbewegung in Flüssen. Betreffend Sedimenttransport wird auf die Untersuchung von Gilbert (1914) und auf die eigene Dissertation hingewiesen, in der die Resultate der klassischen Studie von Paul Du Boys (1847-1924) bestätigt wur- den. Neuere Ergebnisse belegen dabei wiederum den Ansatz von Du Boys, nach dem der Geschiebetrieb gleich ist einer Konstanten mal der Kanalbreite mal dem Sohlgefälle $J$ bei nahezu Normalabfluss, mal der Differenz von $J q$ mit $q$ als Wasserdurchfluss pro Einheitsbreite minus einer Konstanten, eine Beziehung, die jedoch nicht dimensionsgerecht ist. In Kap. 4 wird die Änderung der Sohlenlage im Staubereich besprochen. Da solche Ablagerungen innert weniger Jahre beträchtliche Ausmaße annehmen können und dadurch die Wirkung des Stauraums reduzieren, suchte man schon früh nach Gegenmaßnahmen. Physikalisch schiebt sich von der Stauwurzel her eine Sandbank in Richtung Staustelle, durch welche sich der Stauraum allmählich mit Sedimenten füllt. Eine wichtige Information betrifft die sich dabei einstellende Sohllage nach vollständiger Stauraum-Verlandung. Schoklitsch erläutert ein Konzept, das den Gleichgewichtszustand im Stauraum unter veränderten Wassertiefen respektiert. Kapitel 5 bezieht sich auf Entnahmestellen mit Rücksicht auf die Geschiebebewegung in Flüssen. Nach Abb. 3 entspricht die günstigste Entnahmestelle der Kurvenaußenseite unterstrom des Kolks, da selbst bei mehrstündiger Beaufschlagung mit Sediment kein Geschiebe mehr in die Entnahmestelle gelangte. Diese Versuche bestätigten des Weiteren, dass durch diese Anordnung dem Fluss fast nur Oberflächenwasser entnommen wird, während an der Kurveninnenseite fast nur sandreiches Sohlenwasser 

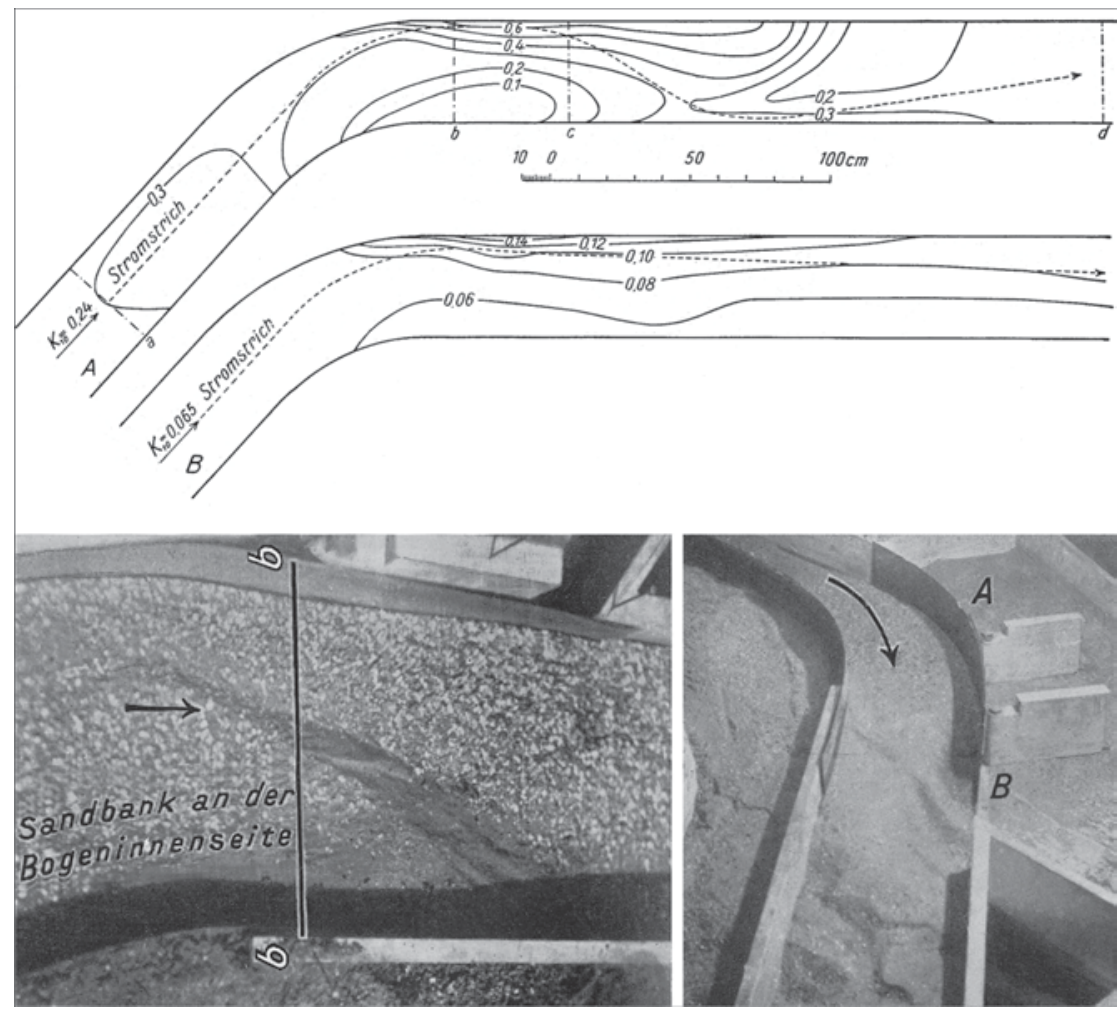

Abb. 3 Geschiebetransport längs einer Kanalkurve bei a) grobem und feinem Sediment, b) Sortierungsvorgang, c) Ansicht vom Unterwasser. (Schoklitsch 1926b)

strömt. In Kap. 6 wird auf die Höhenlage der Einlaufschwelle von Fassungen eingegangen, während sich Kap. 7 mit der Größe des Einlaufquerschnitts beschäftigt. Im Kap. 8 wird dann der Einfluss einer Tauchwand auf die Verlandungserscheinungen diskutiert, während im Kap. 9 die Geometrie des Einlaufbeckens erläutert wird. Die Geschiebebewegung über ein Stauwerk ist Schwerpunkt von Kap. 10, während Kap. 11 sich mit der Spülung des Wehrbereichs beschäftigt. Schließlich folgt in Kap. 12 ein Vergleich zwischen Labormessungen und Naturanlage am Stauwerk Peggau an der Mur. Zusammenfassend darf festgestellt werden, dass Schoklitsch mit dieser Arbeit als einer der Ersten eine Reihe relevanter Probleme der praktischen Geschiebebewegung in Flüssen und Stauwerken anspricht, dass die Resultate aber fallbezogen und deshalb kaum allgemein anwendbar sind. Es soll dabei bedacht werden, dass die Lösung dieser Zweiphasen-Strömungen vor knapp hundert Jahren in den Kinderschuhen steckte, womit Schoklitsch einen wichtigen Beitrag zur vertieften Studie vorlegte. Wie bereits erwähnt, bediente er sich dabei nicht nur der Versuchstechnik, sondern auch der Modellfotografie. Insbesondere augen- fällig ist die Wichtigkeit und Aussagekraft von hydraulischen Modellversuchen, obwohl auf sogenannte Maßstabseffekte (noch) keine Rücksicht genommen wurde. Eine Fortsetzung dieser Arbeit findet sich bei Schoklitsch (1928).

Kleinere Veröffentlichungen von Schoklitsch (1926c, 1926d) betrafen die Statistik der Wasserkraftnutzung in Österreich und sogenannte Energievernichter, ein falscher hydraulischer Ausdruck, da Energie mechanisch nie vernichtet sondern nur in andere Energieformen umgewandelt wird. Dabei wird der Einfluss einer Gegenschwelle in einem Tosbecken hauptsächlich fotografisch analysiert und es werden die internen StrömungsProzesse beschrieben. Der Einfluss der Unterwassertiefe auf die Dissipationsprozesse wird ebenfalls untersucht. Nach diesen noch in Graz abgeschlossenen Arbeiten tritt eine Pause ein, während der sich Schoklitsch auf seine Professur in Brünn vorbereitete sowie das Verfassen zweier Bücher vorantrieb. Schoklitsch (1929b) ist dabei ein Beitrag zum Buch "Ingenieurgeologie", in welchem die Wasserbewegung in Flüssen als $\mathrm{Zu}$ sammenfassung von Schoklitsch (1926b) beschrieben wird. Weiterhin untersucht Schoklitsch (1930a) den Überfallvorgang am rechteckigen Wehr mittels der effektiven Druck- und Geschwindigkeitsverhältnisse.

Ein wichtiges Werk stellt Schoklitsch (1930b) mit dem zweibändigen Buch „Wasserbau“, später „Handbuch des Wasserbaues" vor. Es umfasst in der 1. Auflage 1'185 Seiten und 2'057 Abbildungen. Es ist fast identisch mit der 2. (1950) und der 3. Auflage (1962), weshalb hier die letzte Version betrachtet sei. Das Buch erschien zudem in englischer und in spanischer Sprache. Nach dem Vorwort soll es eine Lücke im damals aktuellen Wasserbau schließen, damit sich der Ingenieur den wichtigsten Fragen stellen konnte. Das Buch besteht aus zwei großen Teilen, nämlich den Grundlagen des Wasserbaus, der Meteorologie, der Hydraulik, der Bodenkunde und der Gewässerkunde, sowie dem praktischen Wasserbau. In der Buchbesprechung wird unter anderem festgehalten, dass „über den derzeitigen Stand der Entwurfsbearbeitung und Ausführung Schoklitsch einen durch Zeichnungen und Lichtbilder sehr instruktiven Überblick in den Gebieten der Wasserversorgung und Ortsentwässerung, der Stauwerke und Entnahmeanlagen, Wasserkraftanlagen, Meliorationen, des Flussbaues und Verkehrswasserbaues gibt. Hervorzuheben ist für das ganze Werk die übersichtliche Gliederung, die klare Herausarbeitung der theoretischen und praktischen Aufgabenstellung und die Erwähnung der Grenzen, bis zu denen unsere Erkenntnisse vorangeschritten sind (...). Die Beschaffung des Werkes kann allen, die sich mit wasserbaulichen Aufgaben beschäftigen, nur empfohlen werden."

Ein Blick in dieses große Werk zeigt die systematische Arbeitsweise und die konsequente Verarbeitung der bereits durchgeführten Untersuchungen durch Schoklitsch. Die einzelnen Kapitel werden dabei durch wichtige Beiträge seiner Fachkollegen ergänzt. Im Gegensatz zu vielen ähnlichen Buchwerken werden viele Arbeiten zitiert, die Beschreibungen sind mit sauberen und klaren Diagrammen, Schemas und Fotos illustriert und durch zahlreiche Tabellen erörtert. Allein das Kapitel Gewässerkunde und Hydraulik besticht durch die riesige Vielfalt der erwähnten Themen, insbesondere natürlich die Abschnitte zum Sedimenttransport, zum Kolkprozess oder zur Grundwasserströmung. Viele Darstellungen zeigen dabei nicht nur den Forscher Schoklitsch, sondern zusätzlich den Ingenieur, welcher durch seine Tätigkeit auch mit der Ingenieurpraxis ein enges Ver- 
hältnis hatte, was sich durch konstruktive Details und Abläufe eines Bauprogramms ergeben. Es darf hier erwähnt werden, dass bis 1930 international kaum ein Buch mit der Vielfalt und der sachlichen Beschreibung wie Schoklitschs „Wasserbau“ existierte. Ähnliche Werke waren auf ein Thema, etwa Staumauern oder Bewässerungen, konzentriert und richteten sich dabei entweder auf den einen oder anderen Aspekt, während Schoklitsch ein Problem möglichst umfassend aber konzis anging. Dabei setzte er im weiten Gebiet Wasserbau neue Horizonte, die in der Folge nur noch von wenigen erreicht wurden. Deshalb darf das Buch sogar noch heute als aktuell im Sinne der Sachauswahl und Beschreibung betrachtet werden, obwohl viele Details im Laufe der Zeit offensichtlich genauer analysiert wurden. Ein ähnliches Werk stellt „Der Grundbau“ dar, das hier aber nicht weiter besprochen werden soll (Schoklitsch 1932a).

Schoklitsch (1932b) bezieht sich, wie so oft, auf eine fotografische Studie zur Umwandlung von hydraulischer Energie etwa im sogenannten Wassersprung. In diesem Basisphänomen der Hydraulik entsteht entweder über oder unter dem Zulaufstrom eine Deck- oder eine Grundwalze, in welcher durch Wirbelbildung die hydraulische Energie hauptsächlich in thermische umgewandelt wird. Schoklitsch stellt richtig fest, dass es sich etwa bei der Deckwalze nicht einfach um einen rotierenden Wasserkörper handelt, sondern dass im Innern der Walze eine Vielzahl kleinerer Wirbel entsteht, die die eigentliche Dissipation bewirken. Diese Wirbel werden durch Abflusszonen verschiedener Geschwindigkeiten gebildet, nämlich der Vorwärtsströmung entlang des Bodens beim Wassersprung und der zugehörigen Rückwärtsströmung entlang der Deckwalze. Weiter beachtlich ist der entsprechende Lufteintrag an diesen Scherschichten, wie man sie beim Wassersprung oder beim Tauchstrahl kennt (Abb. 4).

Die Verkleinerung von Sedimenten in Flüssen wurde durch Schoklitsch (1933) untersucht. Der sogenannte Geschiebeabrieb wurde bereits 1875 vom Deutschen Hermann Sternberg (1825-1885) erforscht. Die von ihm vorgeschlagene exponentielle Abnahme des Geschiebevolumens mit der durchlaufenen Wegstrecke wurde durch Schoklitsch 1914 in seiner Promotionsarbeit bestätigt. In Brünn wurden Versuche in einer umlaufenden Trommel durchgeführt, welche mit Beton und Graniteinlagen ausgekleidet war und eine Geschwindigkeit von bis zu $3 \mathrm{~m} / \mathrm{s}$ erreichte. Das Gesetz von

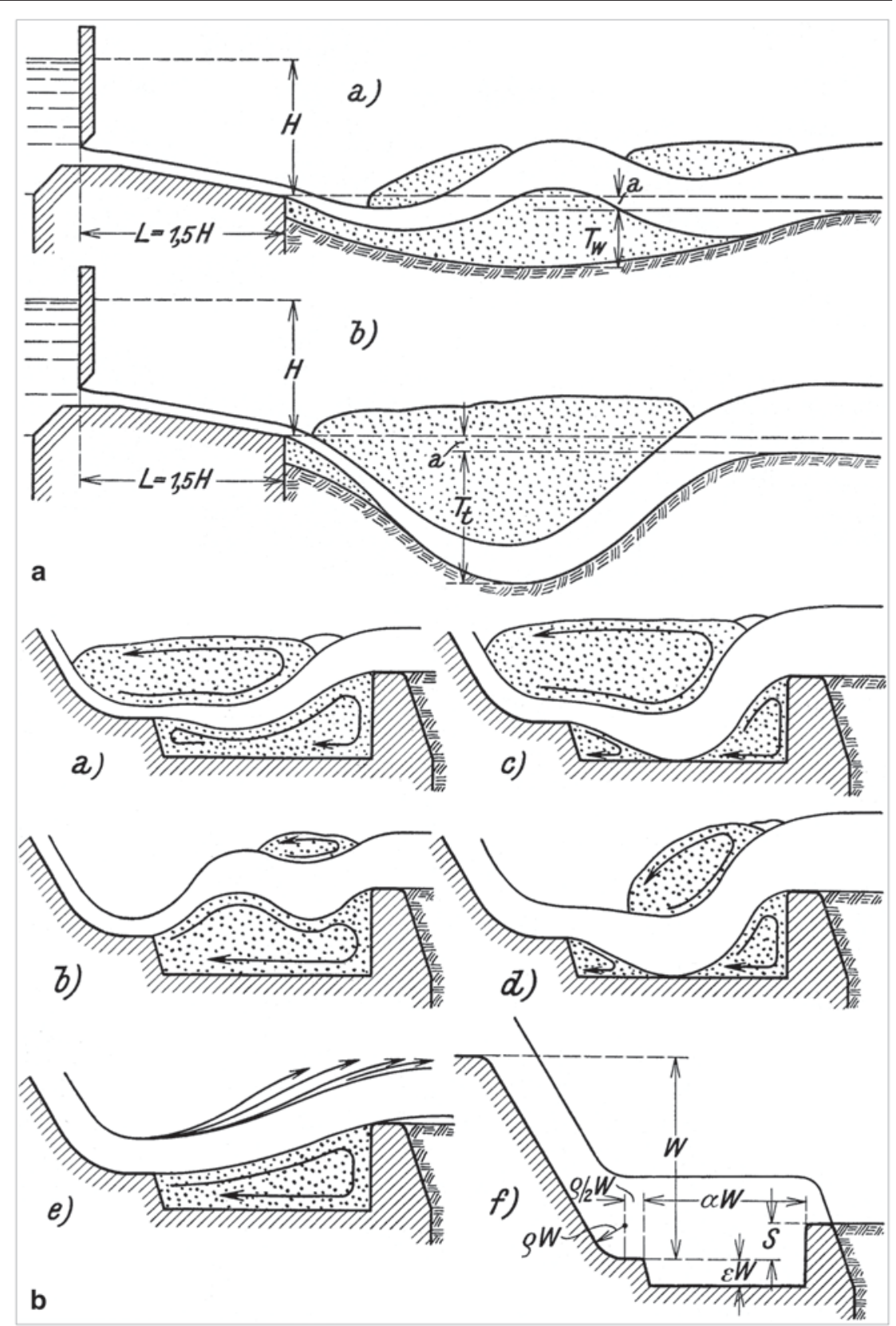

Abb. 4 Abflussprozess längs Wassersprung, bei a) Wellstrahl und Tauchstrahl, b) verschiedene Strahlformen und Definitionsskizze (Schoklitsch 1932b)

Sternberg wurde dahingehend modifiziert, dass die Konstante $c$ nicht nur von der Gesteinsart, sondern zudem von der Geschwindigkeit und von der Beschaffenheit des Unterlagematerials abhängt. Auch in diesem Sektor hat somit Schoklitsch zu Erkenntnissen beigetragen, die eine große Auswirkung auf die Entwicklung einer Flussmorphologie besitzen.

Schoklitsch (1932c) untersuchte experimentell Kolke infolge von Wasserstrahlen. Es wird festgehalten, dass eine Reihe solcher Versuche durchgeführt wurde, dass dabei aber keine Systematik hinsichtlich der Interpretation der Resultate entwickelt worden sei. Die Versuchsanordnung von Schoklitsch bezog sich auf einen durch ein vertikales, scharfkantiges Wehr gebildeten freien Überfallstrahl, der im Unterwasser in ein Wasserpolster mit darunter liegendem Sediment eintaucht. Bezeichnet $Q=q B\left[\mathrm{~m}^{3} / \mathrm{s}\right]$ den Zufluss, $B[\mathrm{~m}]$ die Kanalbreite und $H[\mathrm{~m}]$ die totale Fallhöhe gemessen vom Oberwasserspiegel bis zum Kolkmaximum, so entstehen über dem Kolkboden zwei gegenseitig drehende Wirbel, welche das Geschiebe sowohl in Richtung Wehr als auch ins Unterwasser 


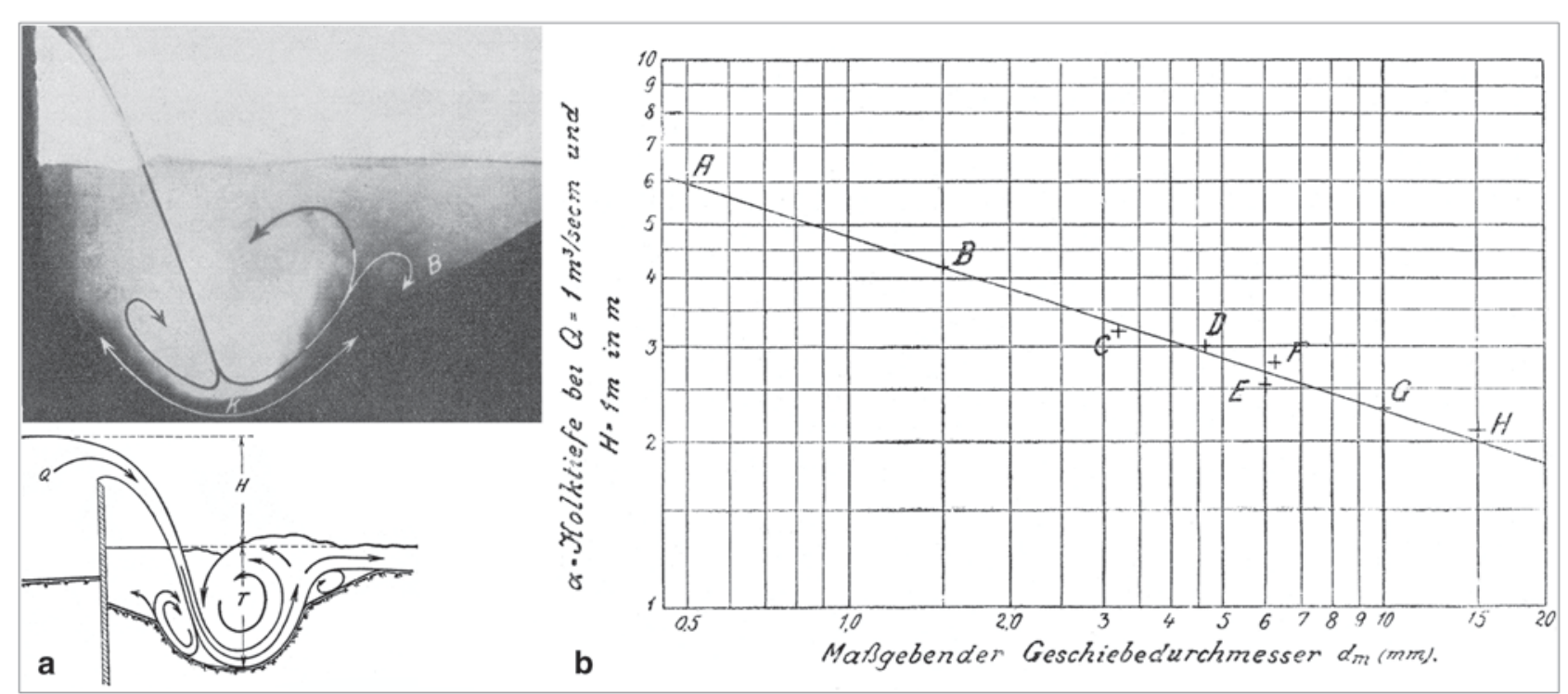

Abb. 5 Kolkentwicklung im Unterwasser eines Überfalls a) Definitionsskizze, b) Datenanalyse. (Schoklitsch 1932c)

bewegen. Nach genügend langer Testzeit stellt sich ein Gleichgewichtszustand ein, bei dem die Kolkgeometrie unveränderlich bleibt. Die Versuche wurden vermutlich wieder von Schoklitsch ausgeführt. Die Resultate ergaben für die Kolktiefe $T$ [m] für den freien und belüfteten Überfallstrahl die folgende, dimensionsfalsche Beziehung (Abb. 5)

$$
T=\left(4.75 / d_{m}^{0.32}\right) H^{0.20} q^{0.57}
$$

Dabei ist $d_{m}$ [mm] nach heutiger Schreibweise $d_{90}$, also das Sediment einer Mischung, bei der $90 \%$ der Körner kleiner sind. Schoklitsch beschreibt zudem den Tauchstrahl und seine Auswirkung auf den Kolkprozess, bespricht die Kornsortierung sowie die sogenannte Abpflästerung. Mit den Resultaten dieser Laborarbeit wurde die erste Kolkformel überhaupt ermittelt; die moderne Kolkforschung setzte dann erst um 1960 ein und ist heute ein Spezialgebiet des Wasserbaus. Weitere Arbeiten zum Kolkproblem finden sich in Schoklitsch (1934a, 1934b, 1935a).

Schoklitsch (1935b) verfasste eine wichtige Arbeit über die Stauraumverlandung und die Kolkabwehr. Im Kapitel Schwemmstoffe und ihre Fortbewegung werden die Eigenschaften und die Bewegungsweise der Geschiebe und deren Abrieb besprochen. Zur rechnerischen Ermittlung des Geschiebetriebs und der Geschiebefracht werden Methoden vorgestellt und anhand von Beispielen illustriert. Das anschließende Kapitel über Schwebstoff beschäftigt sich mit dessen
Entstehung, den physikalischen Eigenschaften, der Verteilung im Abflussquerschnitt und deren Zusammenhang mit dem Wasserdurchfluss. Das Kapitel über die Verlandung von Stauräumen ist eine Fortsetzung von Schoklitsch (1926b). Demnach sollten diese Bereiche nicht nur hinsichtlich des Wassers, sondern eben auch betreffend Geschiebe untersucht werden. Dazu werden wiederum verschiedene Beispiele erläutert, mit denen der Autor durch seine Gutachtertätigkeit zu tun hatte. Schließlich folgt das Kapitel Kolkabwehr und Energievernichtung. Das bereits besprochene Phänomen der Walzenentwicklung und dessen Folge für die Energiedissipation werden in einen größeren Zusammenhang gestellt. Anschließend wird die Kolkentwicklung mit und ohne Abwehr, also Bauwerken, die einen mäßigenden Einfluss auf die Kolktiefe besitzen, diskutiert. Auch hier wird eine Vielzahl von Beispielen sowohl für Strahlkolk als auch für Kolke unterstrom von Tosbecken erläutert. Damit hat Schoklitsch erneut Maßstäbe zur hydraulischen Lösung wichtiger Fragen im $\mathrm{Zu}$ sammenhang mit wasserbaulichen und flussbaulichen Problemen gesetzt. Wie bereits erwähnt wird speziell die Relevanz von hydraulischen Modellversuchen offensichtlich, welche Schoklitsch ausgezeichnet beschreibt und auch entsprechend bildlich dokumentiert (Abb. 6, 9b).

Weitere Arbeiten über den Feststofftransport in Flüssen veröffentlichte Schoklitsch (1934c, 1935e, 1937a, 1938a, 1938b, 1940a, 1940b, 1941, 1942a). Schoklitsch (1937b) publizierte ein besonderes Werk über die Berechnung der Kosten in seinen Fachgebieten, das hier aber infolge dessen zeitlich begrenzter Aussagekraft nicht besprochen wird. Schließlich beschäftigt sich Schoklitsch (1942b) mit sogenannten Schachtüberfällen, die in England und den USA bereits in den 1920er-Jahren angewendet wurden. Kleinere Arbeiten über wasserbauliche, wasserwirtschaftliche und meteorologische Probleme sollen hier nicht diskutiert werden (Schoklitsch 1934d, 1935c, 1935d, 1935f, 1935g, 1935h, 1935i, 1935j, 1936a, 1936b, 1937c, 1937d, 1937e, 1937f, 1937g, 1937h, 1937i, 1937j, 1937k, 1938c, 1938d, 1938e, 1938f, 1939, 1942c, 1942d, 1942e, 1942f). Dies sind meist Zusammenfassungen fremder Beiträge, welche Schoklitsch als Mitarbeiter der deutschen Zeitschrift „Wasserkraft und Wasserwirtschaft" verfasste. Seine letzte Arbeit datiert dabei von 1942, später waren die Kriegsauswirkungen so gravierend, dass an keine seriösen Forschungen mehr gedacht werden konnte. Die Bibliografie wird größtenteils auch durch Poggendorff (1936) erfasst.

\section{Biografie (2)}

Wie von Hager (2015) erwähnt, liegt eine ausführliche Biografie von Schoklitsch vor. Die nachfolgende Kurzbiografie deckt den Zeitraum von 1926 bis 1945 ab. Armin Karl Kurt Schoklitsch wurde 1926 zum Professor für Wasserbau und Grundbau an der Deutschen Technischen Hochschule (DTH) in Brünn ernannt (Anonym 1924, BoehmPilsen 1990, Abb. 7). Er war dabei Nachfolger von Peter Kresnik (1850-1928). In 

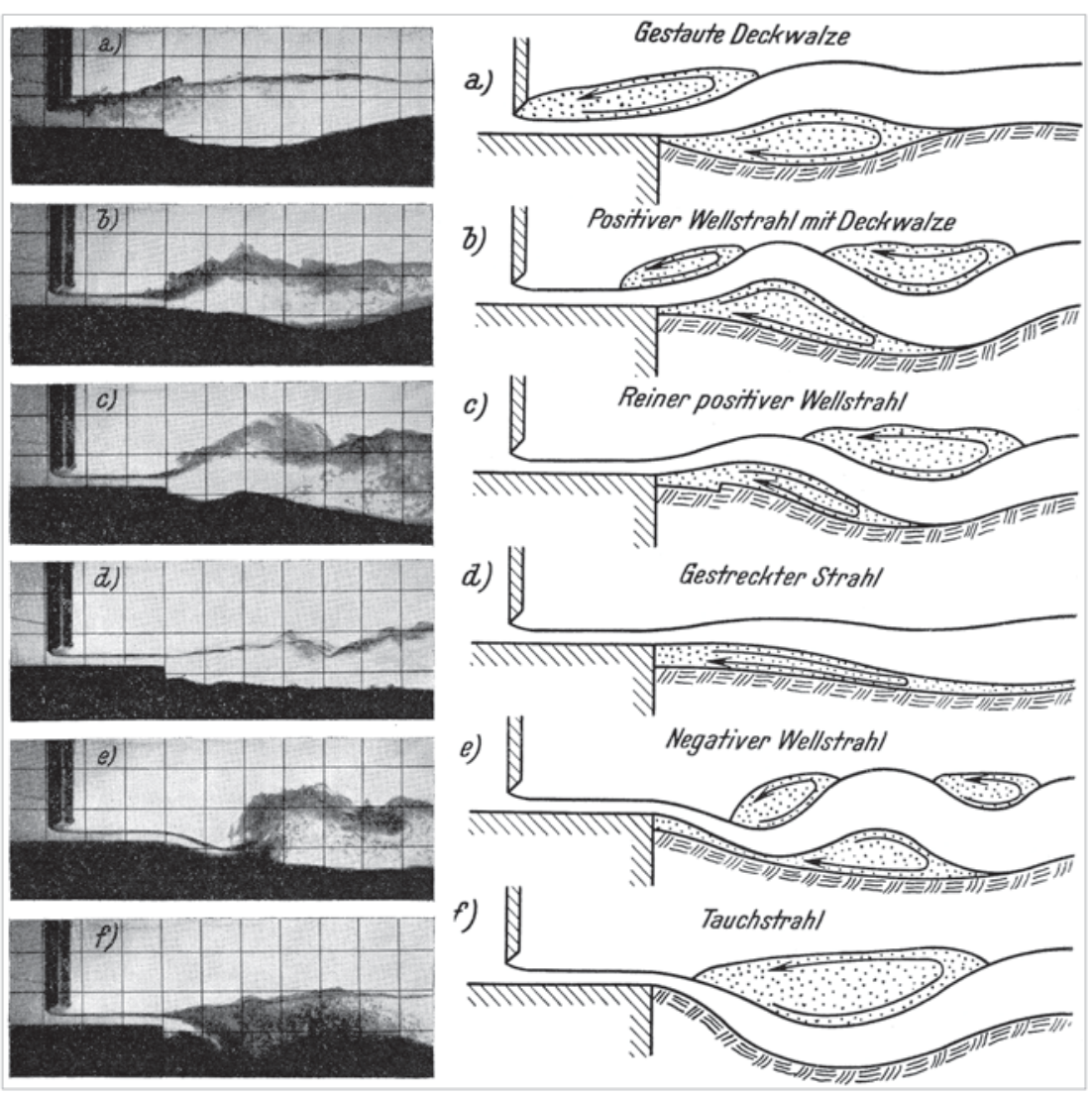

Abb. 6 Strahl- und Kolkformen. (Schoklitsch 1935b)

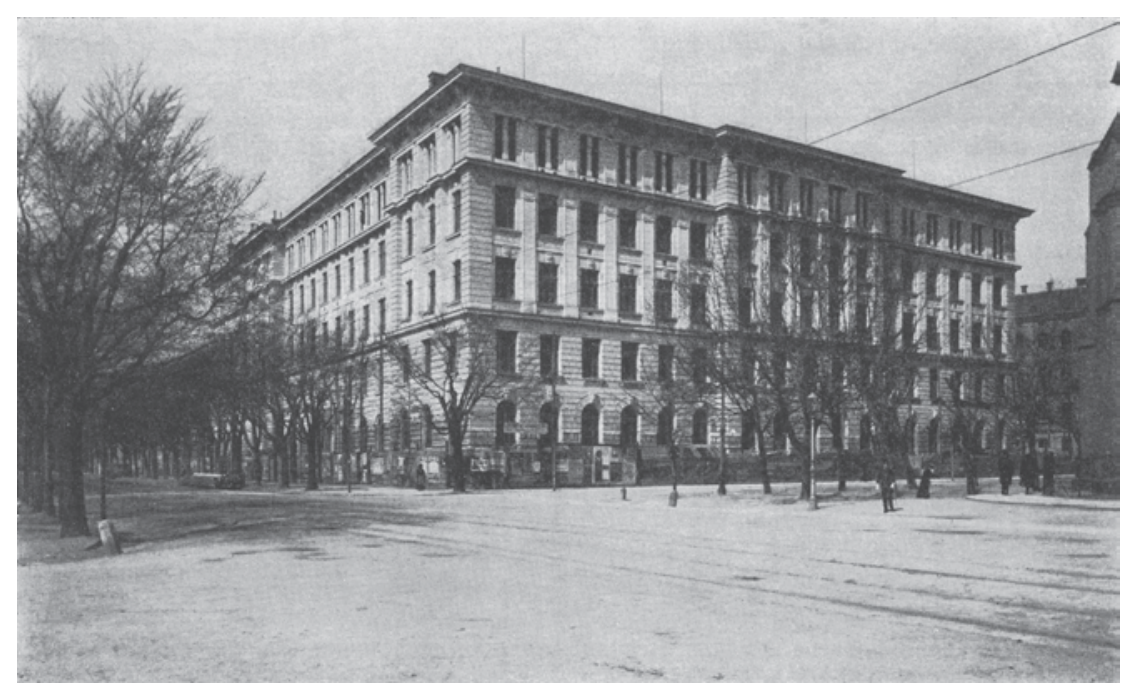

Abb. 7 Neugebäude der DTH Brünn. (Anonym 1924)

einem vom 22. Mai 1925 datierten Brief richtete sich Schoklitsch an das Ministerium für Unterricht und Volkskultur um mitzuteilen, dass er gerne bereit sei, die Lehrkanzel für Wasserbau I in Brünn zu übernehmen. Es folgt neben anderen Bedingungen der Satz: „Ich erlaube mir arbeit nicht zu leisten ist“. Leider ging dieser Wunsch nicht in Erfüllung. Arnold Wintersteiner, nach dem 2. Weltkrieg wohnhaft in Wels, ein ehemaliger Mechaniker und Laborgehilfe an der Lehrkanzel, schrieb: „Unsere Versuchsräume befanden sich in unmittelbarer Nachbarschaft zum Kaplan-Turbinen-Labor. Im Einvernehmen mit meinem Chef [Schoklitsch], der in diesem Jahr im Alter von 81 Jahren in Argentinien verstarb, hatte ich von Fall zu Fall die Instandsetzungsarbeiten im Turbinenlabor zu besorgen, da keine geeignete Fachkraft dem Personalstand beim Lehrstuhl für Turbinenbau angehörte. Der Zustand des Labors war wohl nicht der beste. Die Anlagen standen bis zu den Knöcheln unter Wasser, so dass Laufstege errichtet werden mussten. Unzählige Ratten bevölkerten die Räume. Auch unser Wasserbaulabor ,profitierte von diesen Vierbeinern. Mitunter gab es mit Hilfe anderer Kollegen regelrechte Treibjagden auf diese ungebetenen Gäste der Unterwelt. Das damalige Unterrichtsministerium der zwanziger Jahre hatte für die Anliegen der Forschungsarbeiten an der Deutschen Technik kein allzu großes Interesse, auch wenn es sich um die Wirkungsstätte des genialen Erfinders Kaplan handelte. Ich hatte während meiner 20jährigen Beschäftigung im Keller die gesundheitsschädliche Wirkung an mir selbst beobachten können. Die Einrichtungen an anderen Versuchsanstalten waren unseren gegenüber recht unterschiedlich. Viele Forschungserfolge waren dank der Unterstützung der Industrie im Sudetenland möglich geworden".

Leider liegen fast keine anderen Beschreibungen des Wasserbau-Labors vor. Wie Viktor Kaplan (1876-1934) hat auch Schoklitsch eine gewisse Unterstützung der Industrie erhalten, für welche er ja oft Modellversuche ausführte. Die Bedingungen waren dabei aber schlechter als das in Teil 1 beschriebene Labor an der TH Graz. Trotz misslicher Umstände verstand es Schoklitsch während eines Jahrzehnts, wichtige Forschungsergebnisse aus seinen Untersuchungen zu publizieren. Ab 1935 ließen diese Arbeiten jedoch sichtlich nach, was einerseits Schoklitschs Alter von nahezu 50 Jahren zugeschrieben werden kann, andererseits aber auch fehlenden Mitarbeitern, welche ihm einen Teil der Projekte abnehmen hätten können. Weiter ging Mitteleuropa auf den 2. Weltkrieg zu, also wurden Basisforschungen im nichtmilitärischen Bereich kaum mehr finanziert, was viele deutsche, später aber auch österreichische Universitäten zu spüren be- 


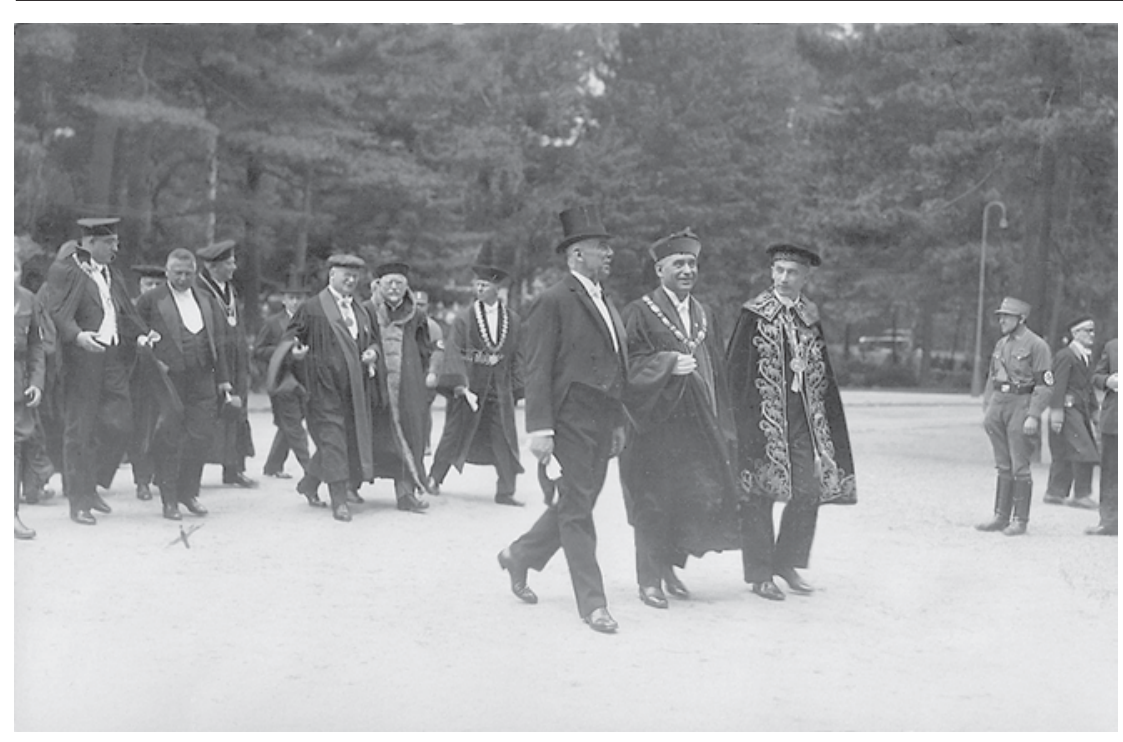

Abb. 8 Schoklitsch (x) als Ehrendoktor der TH Breslau 1936 (Foto: Pietschmann 1999)

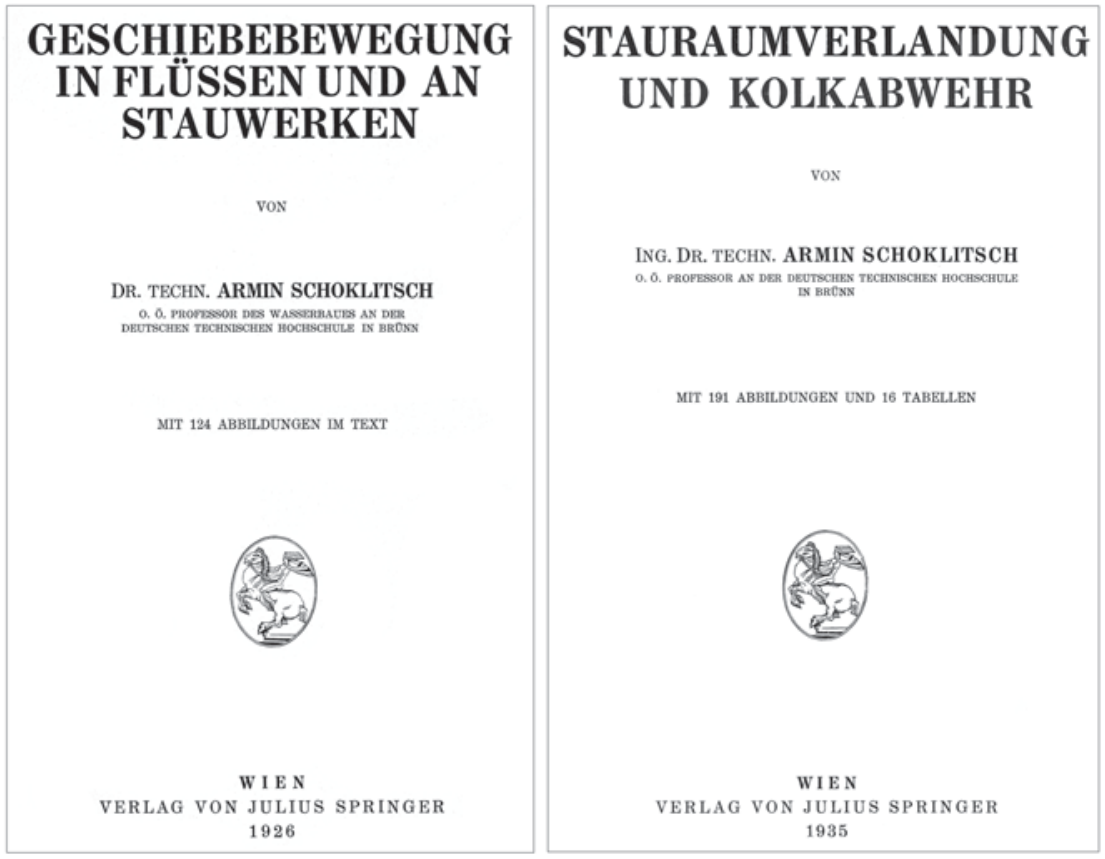

Abb. 9 Titelseiten von a) Schoklitsch (1926), b) Schoklitsch (1935b)

kamen. Bilder von der Kulturausstellung 1928 in Brünn zeigen auch das Wasserbau-Laboratorium, es wurden jedoch nur Fotos von bestehenden Anlagen gezeigt, Laboraufnahmen hingegen fehlen. Im Jahr 1936 wurde Schoklitsch von der TH Breslau (heute Wroclaw in Polen) mit der Würde eines Ehrendoktors der technischen Wissenschaften ausgezeichnet für „seine tiefgehenden Forschungen und wissenschaftlichen Untersuchungen auf dem Gebiete des Grund- und Wasserbaues, insbesondere auf dem der Geschiebebewegung in Gebirgsflüssen und der früher mit einfachen Hilfsmitteln vorwiegend grundlegenden Vorgängen der Strömungsmechanik zugewandt hätten, verlangten die damals (1940) schwierigen Verhältnisse umfangreiche Modellversuche, die den Ausbau des Labors zu einer Versuchsanstalt für alpinen Wasserbau notwendig machten. Anhand von Lichtbildern, die vorwiegend eigenen Versuchen entstammten, erläuterte Schoklitsch grundlegende Ergebnisse seiner Versuche über die Strömungsformen des reinen Wassers, die Bedeutung mitgeführter Stoffe sowie die Relevanz der Laborarbeit im Bereich Eisführung. Es wird festgehalten, dass die Schwebstoffund Geschiebeführung der Flüsse ausgedehnte Versuchsarbeiten verlangen. In enger Zusammenarbeit mit der Praxis führe die Laborarbeit im Wasserbau zudem über die Zweckforschung hinaus in allgemeine Grundlagen des Bauwesens. In solcher Zusammenarbeit bestehe die Hoffnung, auch heute aus dem kleinen bestehenden Labor der TH Graz eine der großen Aufgaben des alpinen Wasserbaus gerecht werdende Versuchsanstalt zu entwickeln. Leider erfüllte sich dieser große Wunsch Schoklitschs nie.

Ab 1942 war Schoklitsch vermutlich amteswegen Leiter des Arbeitskreises Wasserbau und Wasserwirtschaft des Gaus Steiermark. Von November 1944 bis April 1945 diente Schoklitsch zudem als Rektor der TH Graz, nachdem er seit 1942 Prorektor war. Er wurde ebenfalls zum Mitglied der Deutschen Akademie der Wissenschaften in Prag ernannt. Er erwähnt in seinem Lebenslauf, dass seine geplanten Laborarbeiten infolge von Bombenangriffen verunmöglicht wurden. Am 16. April 1945 wurde er zum Volkssturm einberufen, kurz danach aber wieder frei gestellt, um im Auftrag der TH Graz wertvolle Gegenstände und die Rektoratskette nach Liezen zu verlagern, damit diese nicht in russische Hände fielen. Da Schoklitsch bei Kriegsende sowohl Rektor und Parteimitglied war, wurde er von der amerikanischen Besatzung am 6. Juni 1945 abgeholt und in ein Lager bei Oeblarn gebracht, von wo aus er in das (ehemalige) Konzentrationslager Glasenbach bei Salzburg verschoben wurde. Unter den 10'000 Männern waren 4 Rektoren und viele Studenten. Schoklitsch erhielt die Erlaubnis, Vorlesungen abzuhalten und Prüfungen abzunehmen. Nach 26 Monaten wurde das Lager aufgelöst und er ins Bezirksgericht von Graz überstellt, wo das Strafverfahren schließlich nach 28 Monaten eingestellt wurde. Nach kurzer Zeit kam eine Anfrage aus 
England, ob er gewillt wäre, als Professor nach Bombay zu gehen. Da aber zu derselben Zeit eine ähnliche Anfrage aus Tucuman, Argentinien, eintraf entschloss er sich, dieser zuzusagen. Die gestellten Bedingungen umfassten die bezahlte Überfahrt der ganzen Familie bestehend aus 8 Personen und des Haushalts nach Argentinien. Am 5. Februar 1948 ging die Reise vorerst nach Genua, wo auf die Andrea C verschifft wurde. Nach drei Wochen traf die Familie in Buenos Aires ein, von wo die Reise weiter nach Tucuman ging, wo sie der Dekan der Universität empfing. Der Familie wurde eine Villa zur Verfügung gestellt, bis sie ein Haus mieten konnten.

\section{Geschiebetransport}

Das Werk „Wasserbau“ (Schoklitsch 1930b) stieß schnell auf großes Interesse. Der Amerikaner Samuel Shulits (19021973) veröffentlichte eine Arbeit über die Geschiebetransport-Formel von Schoklitsch (Shulits 1935), in welcher neben dieser Beziehung auch jene des Amerikaners Grove K. Gilbert (1843-1918) erwähnt wird. Shulits berichtet, dass dieses Problem Mitte der 1930er-Jahre großes Interesse erweckte, einerseits infolge der vielen in den USA gebauten Talsperren und das dadurch modifizierte Sedimentverhalten in deren Unterwasser, andererseits durch verschiedene Anstrengungen in den Alpenländern Österreich und der Schweiz mit ihren Problemen am Rhein und an der Donau. Die von Schoklitsch (1926b, 1934c) stammenden Arbeiten erlaubten die Ermittlung des Geschiebetransports. Nach einer Zusammenfassung der Resultate von Schoklitsch vergleicht Shulits dessen Resultat mit dem von Gilbert (1914). Shulits seinerseits schlägt dann eine Beziehung vor, welche den Geschiebetransport $G$ in $[\mathrm{lb} / \mathrm{s}]$, also amerikanischen Pfund pro Sekunde in Funktion des Wasserdurchflusses $Q$ minus eines Anspringdurchflusses $B q_{0}$ mit $B$ als Kanalbreite und $q_{0}=0.00532 d / S^{4 / 3}, d$ [in] als Sediment-Durchmesser und $S$ als hydraulisches Gefälle ausdrückt. Es entsteht mit $\gamma$ als spezifischem Sedimentgewicht und $n$ als Sediment-Porosität die Beziehung für uniforme Sedimente (Shulits 1935)

$$
G=\frac{1.39 S^{1.5}}{\gamma(1-n) d^{0.5}}\left[Q-B q_{0}\right]
$$

Es wird festgehalten, dass Gl. (2) prinzipiell einen ähnlichen Aufbau zu jener von Du Boys, resp. Schoklitsch aufweist.
Weiterhin stellt Shulits fest, dass die Gleichung von Schoklitsch Naturmessungen am Oberrhein von Wittmann (1927) gut beschreibt; insbesondere soll die lineare Abhängigkeit $G(Q)$ klar festgestellt worden sein. Weiter wurden Daten von Rudolf Ehrenberger (1882-1956) betrachtet, welcher $G$ an der Donau maß (Ehrenberger 1931, 1932); die Übereinstimmung mit Schoklitsch, resp. Shulits Formeln ist mit Ausnahme eines Punkts wiederum gut. Es wird sogar festgehalten, dass Schoklitschs Formel auch für Geschiebemischungen gilt. Natürlich sind die Limitationen aller Formeln aus heutiger Sicht ungenügend, immerhin konnte aber ein komplexes Problem des Flussbaus nach langer Zeit endlich immerhin näherungsweise beschrieben werden. Als Schlussfolgerung Shulits (1935) folgt: Die Formel von Schoklitsch ist die einzige, welche das Problem umfassend beschreibt, aber das Problem ist so wichtig, dass weitere Untersuchungen angeregt werden. Diese wurden in der Folge an der Versuchsanstalt für Wasserbau der ETH Zürich intensiv angelegt, was schließlich in die heute noch immer aktuelle Formel von MeyerPeter und Müller (1948) mündete.

Abschließend sei noch erwähnt, dass Shulits und Straub (1937) das Werk „Wasserbau" von Schoklitsch mithilfe der Unterstützung des John R. Freeman Trust Estates ins Englische übersetzten. John R. Freeman (1855-1932) initiierte dieses Projekt, um damit amerikanischen Ingenieuren das europäische Wissen, welches dieses Buch so umfassend enthalte, $\mathrm{zu}$ vermitteln. In einem 1930 datierten Brief an Schoklitsch teilt Freeman mit: „Meine Aufmerksamkeit wurde vor etwa zwei Jahren durch Prof. C. E. Bardsley auf ihre Arbeiten gelenkt, welcher mir über ihre erfolgreichen Forschungen trotz teils widerlichen Arbeitsumständen schrieb. Nach der Herausgabe Ihres Buchs Wasserbau wurde mein Interesse geweckt, obwohl meiner ungenügenden Deutschkenntnisse. Ich war überzeugt, dass es sich dabei um das beste Buch auf diesem Sachgebiet handelte; dessen Übersetzung würde die internationale Freundschaft anregen und amerikanische Forschungen unterstützen“. Bekanntlich initiierte Freeman um 1927 die sogenannte Freeman Scholarship, durch die begabte amerikanische Studenten die Unterstützung für einen Besuch an einer europäischen, meist deutschen Hochschule erhielten. Shulits war einer dieser Studenten, welcher in Brünn unter Schoklitsch studierte und im Nachgang dazu eben das Buch
„Wasserbau“ übersetzte. Während seines Besuchs in Brünn im Jahr 1930 erhielt Freeman von Schoklitsch die Übersetzungsrechte. Anschließend begann Shulits mit seiner Arbeit, schloss diese aber leider nicht $a b$, sodass Lorenz G. Straub (1901-1963) das Projekt beendete. Straub war später einer der bekanntesten Hydraulik-Professoren der USA an der University of Minnesota, Minneapolis.

Aus seinem Lebenslauf geht hervor, dass Schoklitsch 1931 von Freeman eine Einladung in die USA erhielt, um insbesondere am Massachusetts Institute of Technology (MIT) Vorträge zu halten und ein Wasserbau-Laboratorium einzurichten. Infolge des plötzlichen Tods von Freeman unterblieb diese Reise jedoch. Schoklitsch klagt weiter, dass durch seine starke Lehrbelastung und die schwierige Lage der deutschen Hochschulen in der Tschechoslowakei die wissenschaftliche Tätigkeit in Brünn stark litt.

\section{Schlussfolgerungen}

Armin Schoklitschs Karriere und sein Werk von 1926 als Professor an der Deutschen Technischen Hochschule in Brünn bis zur Übersiedlung nach Argentinien 1948 werden beschrieben. Insbesondere seine Buchwerke werden analysiert, aber auch die schwierigen Zwischenkriegsjahre in Brünn nachgezeichnet. Durch seine Lehr- und Forschungstätigkeit, aber auch seine Nähe zur wasser- und flussbaulichen Praxis, wurde er Beispiel einer erfolgreichen Persönlichkeit in seinem Fachgebiet, der jedoch mit einem schwierigen politischen Umfeld zu kämpfen hatte. Seine Forschungen im Bereich Sedimenttransport werden speziell diskutiert und in den Kontext seiner Zeit gestellt. Schließlich werden sein Abschied von Europa und die damit verbundenen Schwierigkeiten erläutert. Damit versucht diese Arbeit eine wichtige Persönlichkeit des Wasserbaus und dessen Name der Nachwelt zu erhalten.

\section{Danksagung}

Ich möchte mich bei Frau Dipl.-Ing. Heide Pietschmann, Schoklitschs Enkelin, erneut für die Überlassung persönlicher Fotos bestens bedanken.

\section{Abkürzungsverzeichnis}

W Die Wasserwirtschaft (Österreich)

DW Deutsche Wasserwirtschaft WW Wasserkraft und Wasserwirtschaft 


\section{Literatur}

Anonym (1924): Festschrift der Deutschen Technischen Hochschule in Brünn zur Feier ihres fünfundsiebzigjährigen Bestandes im Mai 1924. DTH: Brünn.

Boehm-Pilsen, J.J. (1990): Die Deutschen Technischen Hochschulen in Prag und Brünn. Österreichische Ingenieur- und Architekten-Zeitschrift 135(5): 232-236.

Ehrenberger, R. (1931): Direkte Geschiebemessungen an der Donau bei Wien und deren bisherige Ergebnisse. W24(34): 581-589.

Ehrenberger, R. (1932): Geschiebemessungen an Flüssen mittels Auffanggeräten und Modellversuche mit letzteren. $W$ 25(33): 449-452; 25(36): 486-490.

Gilbert, G.K. (1914): The transportation of debris by running water. Professional Paper 86 . US Geological Survey: Washington DC.

Hager, W.H. (2015): Armin Schoklitsch: Der Weg zum Wasserbau-Ingenieur (Teil 1). Osterreichische Wasser- und Abfallwirtschaft 67(1-2): 70-75. DOI 10.1007/s00506-015-0212-4

Meyer-Peter, E., Müller, R. (1948): Formulas for bed-load transport. 2nd IAHR Congress Stockholm 2: 1-26.

Poggendorff, J.C. (1936): Schoklitsch, Armin. Biographisch-Literarisches Handwörterbuch 6 2358; 7: 231-232. Verlag Chemie: Leipzig. Schoklitsch, A. (1926a): Über die Durchlässigkeit von Baumaterialien und Sand. W 19(24): 691-695. Schoklitsch, A. (1926b): Geschiebebewegung in Flüssen und an Stauwerken. Springer: Wien. Schoklitsch, A. (1926c): Die Wasserkraftnutzung in Österreich. Wasserkraft-Jahrbuch 1925/26: 22-25. Pflaum: München.

Schoklitsch, A. (1926d): Energievernichter. Die Wasserkraft 21(10): 108-115

Schoklitsch, A. (1928): Kolkbildung und Kolkabwehr unterhalb von Stauwerken. WW 23(15): 217-222.

Schoklitsch, A. (1929a): The Research Laboratory and the researches undertaken therein (Das hydraulische Laboratorium und die darin unternommenen Forschungen). Hydraulic laboratory practice: 309-335, J.R. Freeman, ed. ASME: New York.

Schoklitsch, A. (1929b): Tätigkeit des fliessenden Wassers: Wasserbewegung, Form und Ablagerungen der Flüsse. Ingenieurgeologie: 635-648, K.A. Redlich, K. von Terzaghi, R. Kampe, eds. Springer: Wien.

Schoklitsch, A. (1930a): Zur Berechnung des Ergusses über ein Wehr. $W W$ 25(8): 85-87; 26(2): 21-22.

Schoklitsch, A. (1930b): Der Wasserbau. Springer: Wien.

Schoklitsch, A. (1932a): Der Grundbau: Ein Handbuch für Studium und Praxis. Springer: Wien.

Schoklitsch, A. (1932b): Über die Energievernichtung durch Walzen. W25(16/17): 225-226. Schoklitsch, A. (1932c): Kolkbildung unter Überfallstrahlen. $W 25(24): 341-343$.
Schoklitsch, A. (1933): Über die Verkleinerung der Geschiebe in Flussläufen. Sitzungsberichte Akademie der Wissenschaften Wien, Math-Naturw. Klasse 142(8): 343-366.

Schoklitsch, A. (1934a): Die Kolkbildung an der Oberstromseite von Stauwerken. WW 29(20): 247-248.

Schoklitsch, A. (1934b): Die Kolkabwehr durch eine Wand flussab des Stauwehres. WW 29(23): 279-282.

Schoklitsch, A. (1934c): Der Geschiebetrieb und die Geschiebefracht. WW 29(4): 37-43.

Schoklitsch, A. (1934d): Erfahrungen bei der Betonierung italienischer Talsperren. WW 29(23): 293-294.

Schoklitsch, A. (1935a): Die hydraulische Spülbaggerung und Geländeaufspülung bei Otrokovitz an der March in Mähren. WW 30(16): 191-192. Schoklitsch, A. (1935b): Stauraumverlandung und Kolkabwehr. Springer: Wien.

Schoklitsch, A. (1935c): Druckverlust in grossen Drosselklappen und Johnson-Ventilen. WW 30(5): 59 .

Schoklitsch, A. (1935d): Modellversuche an Hebern. WW 30(7): 82-83.

Schoklitsch, A. (1935e): Über die Schwemmstofführung der Savio und die Verlandung des Stauweihers Quarto im Appenin. WW 30(10): 119.

Schoklitsch, A. (1935f): Dammbruch infolge Grundbruches. WW 30(17): 206-207.

Schoklitsch, A. (1935g): Die spanischen Gewichtsstaumauern über $35 \mathrm{~m}$ Höhe. WW 30(19): 230-232.

Schoklitsch, A. (1935h): Das Elbewehr bei Magdeburg. $W W 30(20): 243-244$

Schoklitsch, A. (1935i): Druckverlust in StahlDruckrohren von Wasserkraftanlagen. WW 30(21) 254-256.

Schoklitsch, A. (1935j): Über die Wandrauhigkeit von Eisenbetondruckrohren und Druckstollen. $W W 30(22): 267$

Schoklitsch, A. (1936a): Durchsickerung unter Stauwerken. WW 31(3): 35

Schoklitsch, A. (1936b): Die ungarischen Bewässerungsanlagen in den Jahren 1932 bis 1934 . WW 31(24): 309-310

Schoklitsch, A. (1937a): Über den Einfluss von Stauwerken auf die Schwemmstofführung der Flüsse. HDI-Mitteilungen 26(15/16): 179-182.

Schoklitsch, A. (1937b): Kostenberechnungen im Wasserbau und Grundbau. Springer: Wien.

Schoklitsch, A. (1937c): Durchfluss durch nahtlose Stahlrohre. WW 32(1): 11

Schoklitsch, A. (1937d): Die Staustufe Vrane an der Moldau. WW 32(2): 23-24

Schoklitsch, A. (1937e): Die Entwicklung des Strassenrohrnetzes und die jährlich auftretenden Schäden in der Wasserversorgungsanlage Budapest. $W W 32(7): 82-83$

Schoklitsch, A. (1937f): Über die Möglichkei der Reiskultur in Mitteleuropa. WW 32(15/16): 183-186.
Schoklitsch, A. (1937g): Kann Entwässerung Klimaänderungen bewirken? WW 32(15/16) 195-196.

Schoklitsch, A. (1937h): Über die Bemessung von Streichwehren. $W W$ 32(18): 209-212; 35(6) 141-142.

Schoklitsch, A. (1937i): Über die Verlandung von Stauräumen. WW 32(20): 240-242.

Schoklitsch, A. (1937j): Gezeitenkraftwerk be Pleasant-Point. WW 32(24): 292.

Schoklitsch, A. (1937k): Die zulässigen Höchstwerte der mittleren Geschwindigkeit in Gerinnen. WW 32(21): 253-254.

Schoklitsch, A. (1938a): Geschiebe- und Schwebforschung. $D W 33(8)$ : 188-192.

Schoklitsch, A. (1938b): Geräte zur Entnahme von Schweb bei der indischen Wasserbauverwaltung. WW 33(17/18): 212-213

Schoklitsch, A. (1938c): Die Ausbildung de Fugen und deren Abdichtung bei neueren italienischen Talsperren. WW 33(3/4): 39-43.

Schoklitsch, A. (1938d): Das neue Kraftwerk be Jons an der Rhône. WW 33(5/6): 69-70

Schoklitsch, A. (1938e): Über den Grundbau in alten Zeiten. WW 33(19/20): 232-236.

Schoklitsch, A. (1938f): Die Wetterregeln de Zigeuner. Meteorologische Zeitschrift 55: 190-192. Schoklitsch, A. (1939): Die Wasserkraftanlagen am unteren Svir in Russland. WW 34(3/4): 41-45.

Schoklitsch, A. (1940a): Geschiebeforschung Voraussetzung geordneter Geschiebewirtschaft. DW 35(11): 351-352.

Schoklitsch, A. (1940b): Zur Versteppung Deutschlands. DW 35(5): 158-159.

Schoklitsch, A. (1941): Siltometer der indischen Wasserbauverwaltung. WW 16(11): 279

Schoklitsch, A. (1942a): Die Schweb- und die Geschiebefracht italienischer Flüsse. WW 37(6): 135-139.

Schoklitsch, A. (1942b): Schachtüberfälle. $W W$ 37(2): $38-40$.

Schoklitsch, A. (1942c): Die Entsandung des Wassers in Kraftanlagen. WW 37(4): 94-95.

Schoklitsch, A. (1942d): Durchfluss von WasserSand-Gemischen und von Lehmaufschwemmungen durch Rohrleitungen. WW 37(10): 235-237. Schoklitsch, A. (1942e): Über Seeanstiche. $W W$ 37(11): 254-257.

Schoklitsch, A. (1942f): Bekämpfung der Frostgefahr bei Wasserkraftanlagen. Praktisches Wissen (17): 218-224

Shulits, S. (1935): The Schoklitsch bed-load formula. Engineering 139(June 21): 644-646; 139(June 28): 687.

Shulits, S., Straub, L.G. (1937): Hydraulic structures. Übersetzung von Wasserbau. ASME: New York.

Wittmann, H. (1927): Der Einfluss der Korrektion des Rheins zwischen Basel und Mannheim auf die Geschiebebewegung des Rheins. $D W$ 22(10): 245-261; 22(11): 283-294; 22(12): 300-310. 